\title{
Improving IVF: is there a limit to our ability to manipulate human biology?
}

\author{
Pasquale Patrizio $^{1}$ (D) $\cdot$ Sherman Silber ${ }^{2}$
}

Received: 6 October 2016 / Accepted: 9 October 2016 / Published online: 28 October 2016

(C) Springer Science+Business Media New York 2016

The overall live birth rates per oocyte remain low (5-10 \%) and have not changed significantly since the start of IVF 38 years ago [1-5]. Why do so many human oocytes and embryos not result in a live birth? Most menstrual cycles in humans do not yield a competent oocyte or a viable embryo. The use of IVF has not changed this biological law of nature $[6,7]$.

IVF can maximize a reproductive cycle, but there is a biological limit imposed on the fraction of human eggs, ovulated naturally or retrieved after stimulation, that can produce a live baby. Even with the application of the best methods available in clinical practice or in embryology laboratories, not every IVF cycle (like not every menstrual cycle in nature [6]) will yield a competent oocyte/ embryo for live birth. We can only strive to maximize pregnancy and live birth rates per transfer by identifying whether in that specific reproductive cycle, there is an embryo of high potential for a birth. However, this is just a matter of selection, not true "improvement." In fact, no selection method will ever increase live birth rates per started

Capsule Modern IVF is allowing couples to have children who otherwise would remain childless. However, despite what appears to be continual improvement in IVF results, there may be an ultimate limit to the reproductive potential of the human oocyte. Properly validated embryo selection methods are keys to higher pregnancy rates per transfer, but not per patient.

Pasquale Patrizio

pasquale.patrizio@yale.edu

Yale University Fertility Center, New Haven, CT 06511, USA

2 Infertility Center of St. Louis, St. Luke's Hospital, St. Louis, MO 63017, USA cycle (due to the intrinsic biological limits of human reproduction).

An approach slowly gaining more acceptance is for all the embryo transfers to be at the blastocyst stage of growth, which means growing the embryos in vitro up to day 5 or 6 of development before transfer. If the embryos arrest before day 5 or 6 , given today's greatly improved laboratory conditions, it probably means that, in that particular cycle, they were not viable and not destined to become a live birth [8]. The recent Cochrane literature supports improved pregnancy rates per transfer with blastocyst as opposed to day 3 transfers [9]. If we were to do all transfers at the blastocyst stage, we would improve the live birth rates when calculated per transfer (since there will be fewer, unnecessary, transfers), although no patients should be deceived into thinking it will improve their overall chance for pregnancy.

There are a number of other methods being promoted for the assessment of embryo viability and competence for a live birth. All are invasive, not completely reliable, costly, and likely result in the non-transfer/discard of some viable embryos. If one proposes pre-implantation genetic screening (PGS) for all blastocysts, there is now sufficient data to demonstrate that PGS is neither sensitive nor specific enough to select all euploid embryos, and there is accumulating data to demonstrate that this could ironically even lower live birth rates [10-16]. Indeed offering sophisticated embryo PGS testing (via array CGH or SNP or qPCR or next-generation sequencing (NGS)) has been shown to be impacted by high rates of trophectoderm embryo mosaicism [11, 13, 14], making the diagnostic accuracy very challenging with the risk of discarding some normal embryos that were incorrectly diagnosed as abnormal. Therefore, the overall pregnancy rate might be decreased rather than increased $[10,14,16]$. But, all these methods are only selection, and not improvement of oocytes or embryos. Recently, the use of PGS has been shown 
to be ineffective also in improving pregnancy rates on intentto-treat analysis of IVF with PGS versus expectant management in patients with recurrent pregnancy loss $[14,16]$.

Another method is the selection of embryos by time-lapse imaging of morphokinetic and morphological parameters, based on the assumption that a continuous observation of embryo's growth can be predictive of embryos with the highest capacity to implant. However, this methodology comes with vastly increased costs and the risk of deselecting embryos able to produce live births. One study, in fact, has reported the birth of healthy children after transfer of blastocysts originating from embryos with abnormal morphokinetic cleavage patterns that should not have been transferred if the time-lapse indications were followed. These authors concluded that only the transfer of viable embryos after 5-6 days of cultured (blastocysts) provides optimal embryo selection [17]. Likewise, another very recent randomized control trial comparing time-lapse selected embryos versus those selected by morphology alone showed that the addition of time-lapse morphokinetic data did not improve clinical reproductive outcomes [18-20].

Therefore, we need to answer the question of what is the easiest, non-invasive, and least expensive way to increase the live birth rate per transfer without lowering the live birth rate per patient. We propose just very simply the adoption of exclusive blastocyst-stage transfers (day 5 or 6 embryos) as a method that will immediately improve the pregnancy rates per transfer. Blastocyst transfers are not associated with any likelihood of discarding normal embryos that were wrongly diagnosed as abnormal (as with PGS because of trophoblast mosaicism, or judged abnormal as per morphokinetic parameters). By utilizing only blastocyst-stage embryos for transfer, the live birth and pregnancy rates per transfer and implantation rates across all ages will quickly surge.

Clinicians may worry about the patient's reaction to not having a transfer. With proper counseling, patients will be thankful for knowing early in the process that the cycle was not successful because there were no transferable embryos, if none developed to the blastocyst stage. This will save unnecessary emotional stress, reduce "false hopes," avoid unnecessary supplementation of progesterone, reduce costs, and allow patients to move sooner to another cycle or to alternative plans. Another question that cannot be answered at this time with unequivocal certainty is whether some patients may still benefit from day 3 embryo transfers instead of day 5 , on the assumption that the laboratory conditions might impair the further development of an embryo. If there is any doubt in the physician's mind for a particular group of patients (particularly those with three or fewer embryos), then they could opt for a day 3 transfer for the first cycle of IVF, and if it fails then, blastocyst culture would be used for selection in a subsequent cycle. As long as viable embryos are not mistakenly discarded as with the current imprecise trophectoderm biopsy results and morphokinetic parameters, the shorter time to pregnancy will be of benefit.

In summary, despite today's greatly improved laboratory conditions and the individualization of stimulation protocols, the process of IVF is still inefficient with low live birth rates per embryos produced and per oocytes retrieved. This is because the majority of human oocytes harvested and the majority of embryos produced are deficient in developmental competence, due, in part, to genetic abnormalities of gametic or embryonic origin. The ability to confidently identify gametes and embryos with the greatest reproductive potential would not improve overall live birth rates, but it would improve the success rate per transfer and lessen the agony of waiting for what turns out to be a negative pregnancy test.

Of course, better than improved embryo selection is to actually improve live birth rates per oocyte and per stimulated cycle. A carefully conducted "big data" multivariate regression analysis has ironically linked high FSH overdosage in ovarian stimulation to lower success [21]. Tampering too much with nature in this case actually has lowered IVF success. So, perhaps the best advance now for IVF is to take a step backward rather than forward, recognizing the inherited deficiency of the human oocyte and just maximizing natural selection accuracy.

\section{References}

1. Kovalevsky G, Patrizio P. High rates of embryo wastage with use of assisted reproductive technology: a look at the trends between 1995 and 2001 in the United States. Fertil Steril. 2005;84:325-30.

2. Patrizio P, Bianchi V, Lalioti MD, Gerasimova T, Sakkas D. High rate of biological loss in assisted reproduction: it is in the seed, not in the soil. RBM Online. 2007;14:92-5.

3. Patrizio P, Fragouli E, Bianchi V, Borini A, Wells D. Molecular methods for selection of the ideal oocyte. RBM Online. 2007;15: 346-53.

4. Patrizio P, Sakkas D. From oocyte to baby: a clinical evaluation of the biological efficiency of in vitro fertilization. Fertil Steril. 2009;91:1061-6.

5. Martin JR, Bromer JG, Sakkas D, Patrizio P. Live babies born per oocyte retrieved in a subpopulation of oocyte donors with repetitive reproductive success. Fertil Steril. 2010;94:2064-8.

6. Hertig TA. Human trophoblast: normal and abnormal. Am J Clin Pathol. 1967;47(3):249-68.

7. Opitz JM, Fitzgerald JM, Reynolds JF, Lewin SO, Daniel A, Ekblom SL, et al. The Montana fetal genetic pathology program and a review of prenatal death in human. Am J Med Genet Suppl. 1987;3:93-112.

8. Adler A, Lee HL, McCulloh DH, et al. Blastocyst culture selects for euploid embryos: comparison of blastomere and trophectoderm biopsies. RBM Online. 2014;28:485-91.

9. Glujovsky D, Blake D, Farquhar C, Bardach A. Cleavage stage versus blastocyst stage embryo transfer in assisted reproductive technology. Cochrane Database Syst Rev. 2012;7, CD002118. 
10. Kushnir VA, Darmon SK, Albertini DF, Barad DH, Gleicher N. Effectiveness of in vitro fertilization with preimplantation genetic screening: a reanalysis of the United States assisted reproductive technology data 2011-2012. Fertil Steril. 2016;106(1):75-9.

11. Greco E, Minasi MG, Fiorentino F. Healthy babies after intrauterine transfer of mosaic aneuploid blastocysts. N Engl J Med. 2015;373: 2089-90.

12. Orvieto R, Shuly Y, Brengauz M, Feldman B. Should preimplantation genetic screening be implemented to routine clinical practice? Gynecol Endocrinol. 2016;32(6):506-8.

13. Scott RT, Galliano D. The challenge of embryonic mosaicism in preimplantation genetic screening. Fertil Steril. 2016;105(5):1150-2.

14. Gleicher N, Vidali A, Braverman J, Kushnir VA, Barad DH, Hudson $\mathrm{C}$, et al. Accuracy of preimplantation genetic screening (PGS) is compromised by degree of mosaicism of human embryos. Reprod Biol Endocrinol. 2016;14(1):54.

15. Kang HJ, Melnick AP, Stewart JD, Xu K, Rosenwaks Z. Preimplantation genetic screening: who benefits? Fertil Steril. 2016;106(93):597-602.

16. Orvieto R, Gleicher N. Should pre implantation genetic screening (PGS) be implemented to routing IVF practice? J Assist Reprod Genet 2016.
17. Murugappan G, Shahine LK, Perfetto CO, Hickok LR, Lathi RB. Intent to treat analysis of in vitro fertilization and preimplantation genetic screening versus expectant management in patients with recurrent pregnancy loss. Hum Reprod. 2016;31:1668-74.

18. Stecher A, Vanderzwalmen P, Zintz M, Wirleitner B, Schuff M, Spitzer D, et al. Transfer of blastocysts with deviant morphological and morphokinetic parameters at early stages of in-vitro development: a case series. RBM Online. 2014;28(4):424-35.

19. Goldman LR, Goldberg J, Falcone T, Austin C, Desai N. Does the addition of time-lapse morphokinetics in the selection of embryos for transfer improve pregnancy rates? A randomized controlled trial. Fertil Steril. 2016;105(2):275-85.

20. Wu YG, Lazzaroni-Tealdi E, Wang Q, Zhang L, Barad DH, Kushnir VA, et al. Different effectiveness of closed embryo culture system with time-lapse imaging in comparison to standard manual embryology in good and poor prognosis patients: a prospectively randomized pilot study. Reprod Biol Endocrinol. 2016;14(1):49.

21. Baker VL, Brown MB, Luke B, Smith GW, Ireland JJ. Gonadotropin dose is negatively correlated with live birth rate: analysis of more than 650,000 assisted reproductive technology cycles. Fertil Steril. 2015;104:1145-52. e5. 